\title{
Efectos del fósforo de un efluente cloacal sobre la morfología interna y externa de Eichhornia crassipes (Mart. Solms) en un humedal artificial
}

\author{
M. V. Campanella ${ }^{1}$, H. Hadad ${ }^{2,3}$, M. A. Maine ${ }^{2}$ \& R. Markariani ${ }^{1}$ \\ ${ }^{1}$ Facultad de Humanidades y Ciencias, UNL. Ciudad Universitaria Paraje "El Pozo" (3000) Santa Fe, \\ Argentina. \\ ${ }^{2}$ Consejo Nacional de Investigaciones Científicas y Técnicas (CONICET). Química Analítica, Facultad de \\ Ingeniería Química, UNL. Santiago del Estero 2829 (3000) Santa Fe, Argentina. T. E.: 54 - 0342 - 4571164 \\ Int. 2515. \\ 3hhadad@fhuc.unl.edu.ar
}

\section{RESUMEN}

Se compararon las variaciones morfológicas que presentó Eichhornia crassipes (Mart. Solms) a la entrada y salida de un humedal construido para tratar un efluente cloacal. Se determinó la concentración de fósforo en el agua y en las plantas. Se midió la altura de las plantas, la longitud de las raíces y la biomasa. Para estudiar la morfología interna de la raíz se calculó el área transversal de la raíz (ATR), de la médula (ATM) y de los vasos metaxilemáticos tardíos (ATV) y el área total del metaxilema (ATVt) por sección.

Las diferentes concentraciones de fósforo presentes en el efluente cloacal, a la entrada y salida del humedal, produjeron variaciones en la morfología externa e interna de E. crassipes. La concentración de fósforo en los tejidos mostró una relación directa con la concentración de fósforo en el agua. En las plantas de la entrada se observó una mayor altura, menor longitud y biomasa de las raíces en comparación con las plantas de la salida. En las plantas de la entrada se observó un aumento de los valores de ATM y ATV y ATVt, comparadas con las plantas de la salida. La variación en los parámetros morfológicos internos se debió probablemente al aumento del área transversal de la raíz.

Palabras claves: Eichhornia crassipes - Fósforo - variación morfológica - humedal artificial.

\section{ABSTRACT}

The present study compares the morphological variation of Eichhornia crassipes (Mart. Solms) between the inlet and outlet zones of a wetland constructed for the treatment of a sewage effluent. The phosphorus concentration in water and plants was determined. Plant height, root length and dry biomass were measured. The areas of cross-sectional whole root (ATR), stele (ATM), large metaxylematic vessels (ATV) and the total metaxylematic vessels (ATVt) were calculated.

The different phosphorus concentrations registered at the inlet and outlet zones of the constructed wetland, induced internal and external morphological changes in E. crassipes. Phosphorus concentration in tissues was positively correlated with phosphorus concentrations in water. The inlet zone plants were taller and they had shorter roots and less proportion of root dry weight in comparison with the outlet zone plants. The inlet zone plants increased the ATM, ATV and ATVt values, in comparison with the outlet zone plants. The variation in the internal morphological parameters was probably due to the increase of the cross-sectional whole root.

Keywords: Eichhornia crassipes - Phosphorus - morphological change - constructed wetland.

\section{INTRODUCCIÓN}

La mayoría de las investigaciones sobre humedales han sido realizadas para la depuración de efluentes cloacales y agrícolas, donde los conta- minantes a eliminar fueron el fósforo y el nitrógeno (Athie \& Cerri, 1987; Sedlack, 1991; Weisner et al., 1994; Adler et al., 1996; Perdomo et al., 2000; Merlin et al., 2002; Ansola et al., 2003). Se ha demostrado que en un humedal, la 
desnitrificación es el principal mecanismo de eliminación de nitrógeno (Saunders \& Kalff, 2001), mientras que el fósforo sólo puede ser removido del agua por adsorción de los sedimentos o absorción de las plantas acuáticas. En el humedal estudiado, se demostró que $E$. crassipes fue la responsable de la remoción del $80 \%$ del $\mathrm{P}$ (Maine et al., 2005) y menos del $10 \%$ del nitrógeno inorgánico (Maine et al., 2004).

El fósforo puede alterar el desarrollo de la planta de dos formas, una es actuando como nutriente estimulando la producción de biomasa aérea, y otra es funcionando como una señal que regula los cambios en la arquitectura de la raíz (López-Bucio et al., 2002). Dichos cambios pueden afectar profundamente la capacidad de la planta para tomar nutrientes y agua. Una primera adaptación a las bajas disponibilidades de fósforo involucra cambios en el desarrollo postembrionario del sistema radicular. Esto incluye alteraciones en la longitud total de la raíz, en el alargamiento de los pelos radiculares y en la formación de raíces laterales (Dinkelaker et al., 1995; Bates \& Lynch, 1996; Borch et al., 1999). Esto sugiere que la plasticidad morfológica es un importante mecanismo adaptativo para adquirir nutrientes (Hutchings \& de Kroon, 1994).

Investigaciones relacionadas con el crecimiento y la producción de biomasa de E. crassipes en humedales naturales demostraron que la biomasa aumenta en función de las concentraciones de nutrientes, creciendo en mayor medida la parte aérea en comparación con la raíz (Lallana \& Kieffer, 1988). Por otro lado, la biomasa de las raíces es mayor en las plantas cultivadas en aguas pobres en nutrientes (Reddy \& Sutton, 1984). Según Bloom et al. (1985) las plantas tienden a responder a la limitación de los recursos asignando una mayor proporción de su biomasa a aquellos órganos implicados en la captura de los mismos.

En relación con las variaciones en la morfología interna, Harvey y van den Driessche (1999) encontraron que los vasos xilemáticos de las raíces del álamo tendieron a aumentar su diámetro en respuesta a niveles altos de nutrientes en el medio.

El objetivo del trabajo fue determinar el efecto del fósforo sobre la morfología tanto externa como interna de plantas de E. crassipes localizadas a la entrada y a la salida de un humedal construido para el tratamiento de un efluente cloacal.

\section{MATERIAL Y MÉTODOS}

\section{Sitio de estudio}

El humedal donde se llevó a cabo el estudio se localiza en la planta de la industria metalúrgica Bahco Argentina S.A. de la ciudad de Santo Tomé, Santa Fe, Argentina ( $31^{\circ} 40^{\prime} 01.9^{\prime \prime} \mathrm{S}$, $60^{\circ} 47^{\prime} 06.9$ " O).

El humedal es del tipo de flujo superficial. Las dimensiones son de $50 \mathrm{~m}$ de largo por $40 \mathrm{~m}$ de ancho y profundidad de 50 a $80 \mathrm{~cm}$, con un tiempo de residencia hidráulica estimado entre 7 y 12 días. Presenta un tabique central, paralelo al sentido de circulación del efluente, el cual divide al humedal en dos secciones de igual superficie y obliga al efluente a recorrer el doble de distancia.

El fondo del humedal está impermeabilizado con 5 capas de bentonita compactadas (profundidad aproximada de $60 \mathrm{~cm}$ ), logrando una conductividad hidráulica de $10^{-7} \mathrm{~m} \mathrm{~s}^{-1}$ para evitar la contaminación del agua subterránea. Sobre la bentonita compactada, se colocó suelo del área de excavación hasta una altura de un metro.

El efluente, después de atravesar el humedal, es conducido por un canal hacia una laguna de 1 hectáreas.

Se recolectaron 50 plantas al azar, tanto a la entrada como a la salida del humedal, en diciembre de 2002. Al mismo tiempo, se recogió agua de los mismos sitios en botellas de plástico, colocándolas en conservadoras de frío portátiles. Inmediatamente, las muestras de agua se llevaron al laboratorio y se efectuaron los análisis químicos (Vollenweider, 1974; APHA, 1998). A fin de obtener parámetros de comparación como testigo, se recolectaron plantas de $E$. crassipes y agua en una laguna cercana a la ciudad de Santa $\mathrm{Fe}$, Argentina (31 38' 02.4" S, 60 40' 03.1" O). Las plantas se colocaron en bolsas de plástico y se llevaron inmediatamente al laboratorio. 
Tabla 1. Parámetros fisicoquímicos, concentraciones de fósforo y especies nitrogenadas y relación N:P del agua muestreada a la entrada y salida del humedal artificial y en un ambiente natural testigo (Media \pm desviación estándar). Physico-chemical parameters, phosphorus and inorganic nitrogen concentrations, and N:P ratio of the sampled water at the inlet and outlet zones of the constructed wetland and in a control natural environment (Mean \pm standard deviation).

\begin{tabular}{cccc}
\hline Parámetros & Entrada & Salida & Testigo \\
\hline $\mathrm{pH}$ & $7.59 \pm 0.02$ & $7.52 \pm 0.02$ & $6.97 \pm 0.02$ \\
Conductividad $(\mathrm{mmhos} / \mathrm{cm})$ & $480 \pm 10$ & $470 \pm 10$ & $164 \pm 10$ \\
Fósforo $(\mathrm{P})(\mathrm{mg} / \mathrm{l})$ & $0.240 \pm 0.02$ & $0.026 \pm 0.004$ & $0.05 \pm 0.01$ \\
Nitrato $\left(\mathrm{NO}_{3}^{-}\right)(\mathrm{mg} / \mathrm{l})$ & $10.7 \pm 0.3$ & $5.3 \pm 0.3$ & $1.72 \pm 0.3$ \\
Nitrito $\left(\mathrm{NO}_{2}^{-}\right)(\mathrm{mg} / \mathrm{l})$ & $0.121 \pm 0.05$ & $0.013 \pm 0.003$ & $0.006 \pm 0.05$ \\
Amonio $\left(\mathrm{NH}_{4}^{+}\right)(\mathrm{mg} / \mathrm{l})$ & $1.98 \pm 0.02$ & $0.98 \pm 0.02$ & $0.65 \pm 0.02$ \\
Nitrógeno inorgánico $(\mathrm{N})(\mathrm{mg} / \mathrm{l})$ & $3.99 \pm 1.32$ & $2.38 \pm 0.835$ & $0.894 \pm 0.086$ \\
$\mathrm{~N}: \mathrm{P}$ & 16.6 & 91.5 & 17.9 \\
\hline
\end{tabular}

\section{Análisis químicos}

Sobre tres réplicas de las muestras de agua se efectuó la determinación de fósforo (Murphy \& Riley, 1962), $\mathrm{NH}_{4}^{+}$(Electrodo selectivo de amonio, Orion ion plus 9512BN), $\mathrm{NO}_{3}^{-}$(Electrodo selectivo de Nitrato, Orion ion plus 9700N) y $\mathrm{NO}_{2}^{-}$por método colorimétrico (APHA, 1998). La conductividad se midió utilizando un conductímetro YSI 33 y el pH usando un electrodo de vidrio Orion. El fósforo total en tejidos vegetales se determinó sobre dos réplicas, previa digestión ácida $\left(\mathrm{HCl} / \mathrm{NO}_{3} \mathrm{H} / \mathrm{HClO}_{4}\right)$ según Murphy \& Riley (1962).

\section{Estudio de la morfología externa}

Se describió la morfología externa utilizando como parámetros: longitud de raíces, altura de plantas y peso seco de la parte aérea y de las raíces por planta.

Para estimar el peso seco por planta, los vegetales recolectados se lavaron y separaron en sus partes aéreas (hojas) y sumergidas (raíces), colocándolas en estufa a $105^{\circ} \mathrm{C}$ hasta peso constante (Vollenweider, 1974; APHA, 1998). A partir del peso seco, se calculó la relación parte aérea/raíz.

\section{Estudio de la morfología interna}

Para el estudio de la morfología interna de la raíz se recolectaron segmentos de 3 a $4 \mathrm{~cm}$ de longitud de la zona media de la misma, los cuales inmediatamente se fijaron en formol $4 \%$. Transcurridas $48 \mathrm{~h}$, se colocaron en etanol $70 \%$ para su conservación.

Se realizaron preparados frescos cortando las raíces transversalmente a mano alzada con hojas de afeitar nuevas (D’Ambrogio de Argüeso, 1986). Los cortes transversales de raíz se colorearon con Azul de Toluidina (Wahl et al., 2001).

Las secciones se examinaron en un microscopio óptico Olympus (4X, 10X y 40X). Se midieron los diámetros de la raíz, de la médula y de los vasos metaxilemáticos tardíos con un ocular micrométrico. Para calcular los valores respectivos del área transversal de la raíz (ATR), de la médula (ATM) y de los vasos metaxilemáticos tardíos (ATV), se utilizaron las fórmulas para el cálculo del área de un círculo. Cuando fue necesario, se utilizaron las fórmulas para el cálculo del área de una elipse o de una parábola.

Se contó el número de vasos metaxilemáticos tardíos por sección y se calculó el área total del metaxilema (ATVt) sumando las áreas de todos los vasos por corte.

\section{Análisis estadísticos}

Los cálculos se llevaron a cabo utilizando el software Statgraphics Plus 3.0. Debido a que las poblaciones no presentaron una distribución normal, se aplicaron test no paramétricos y se realizaron gráficos de caja y bigote, utilizando la mediana como medida de tendencia central y el rango intercuartílico (25 y $75 \%$ ) como medida de 
Tabla 2. Concentraciones de fósforo en tejidos vegetales (parte aérea y raíz) de E. crassipes a la entrada y salida del humedal artificial y en un ambiente natural testigo (Media \pm desviación estándar). Phosphorus concentrations in E. crassipes tissues (aerial part and root) at the inlet and outlet zones of the constructed wetland and in a control natural environment (Mean \pm standard deviation).

\begin{tabular}{cccc}
\hline Concentración de $\mathbf{P}(\mathbf{m g} / \mathbf{g}$ peso seco) & Entrada & Salida & Testigo \\
\hline Parte aérea & $5.50 \pm 0.33$ & $2.61 \pm 0.18$ & $3.47 \pm 0.13$ \\
Raíz & $4.47 \pm 0.27$ & $2.19 \pm 0.14$ & $2.92 \pm 0.06$ \\
\hline
\end{tabular}
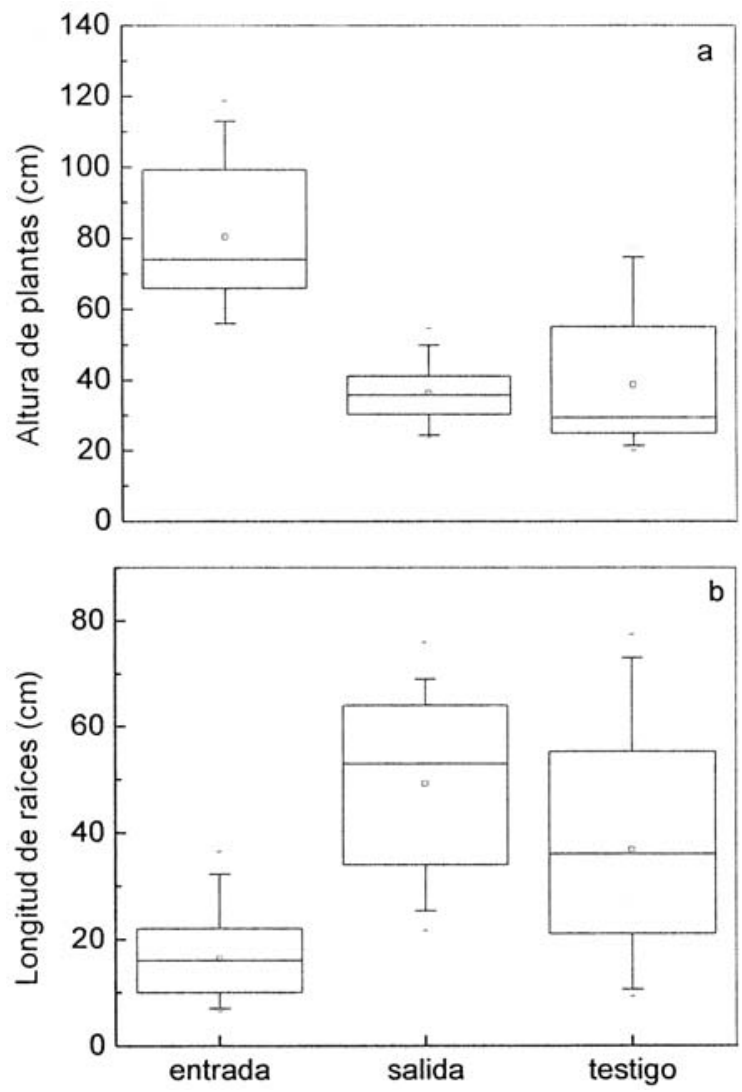

Figura. 1. Gráficos de caja y bigote de la altura de las plantas (a) y longitud de las raíces (b) de E. crassipes en los diferentes sitios de muestreo. Box and wisker plot of plant height (a) and root length (b) of E. crassipes in the diferent sampling sites.

variabilidad. Se aplicó el análisis de KruskalWallis para testar las diferencias entre los parámetros medidos de las plantas de la entrada, de la salida del humedal y las testigo (Walpole et al., 1999). Si se observaban diferencias estadísticamente significativas, se utilizó el test de Wilcoxon para comparar los tratamientos entre sí. En todas las comparaciones se utilizó un nivel de $\mathrm{p}<0.05$.

\section{RESULTADOS}

\section{Análisis químicos}

Algunos parámetros fisicoquímicos y la concentración de fósforo y especies nitrogenadas medidas en el agua a la entrada y a la salida del humedal construido, y del punto de muestreo testigo se representan en la Tabla 1. Los datos evidencian la disminución de las concentraciones de los nutrientes disueltos del efluente después de atravesar el humedal, demostrando la eficiencia de dicho sistema como depurador del efluente. El humedal mostró una eficiencia del $96 \%$ en la retención del fósforo disuelto. No se observó lo mismo para las especies nitrogenadas, ya que los porcentajes de remoción fueron sólo de alrededor del $50 \%$.

Las concentraciones de fósforo en la parte aérea y raíces de $E$. crassipes presentan una relación directa con la concentración de fósforo en agua, observándose la mayor concentración de fósforo en la parte aérea (Tabla 2).

\section{Morfología externa}

La biomasa aérea y la altura fueron significativamente mayores en las plantas de la entrada del humedal en comparación con las plantas de la salida y testigo, probablemente debido a que a la entrada, las concentraciones de nutrientes en el efluente eran altas (Figs. 1a, 2a). Entre las plantas de la salida y testigo no se encontraron diferencias estadísticamente significativas.

Para la longitud de raíces se registraron diferencias estadísticamente significativas entre los tres sitios de estudio. La longitud de raíces fue menor cuanto mayor fue la concentración de fósforo, siguiendo la relación Salida $>$ Testigo $>$ Entrada (Fig. 1b). 

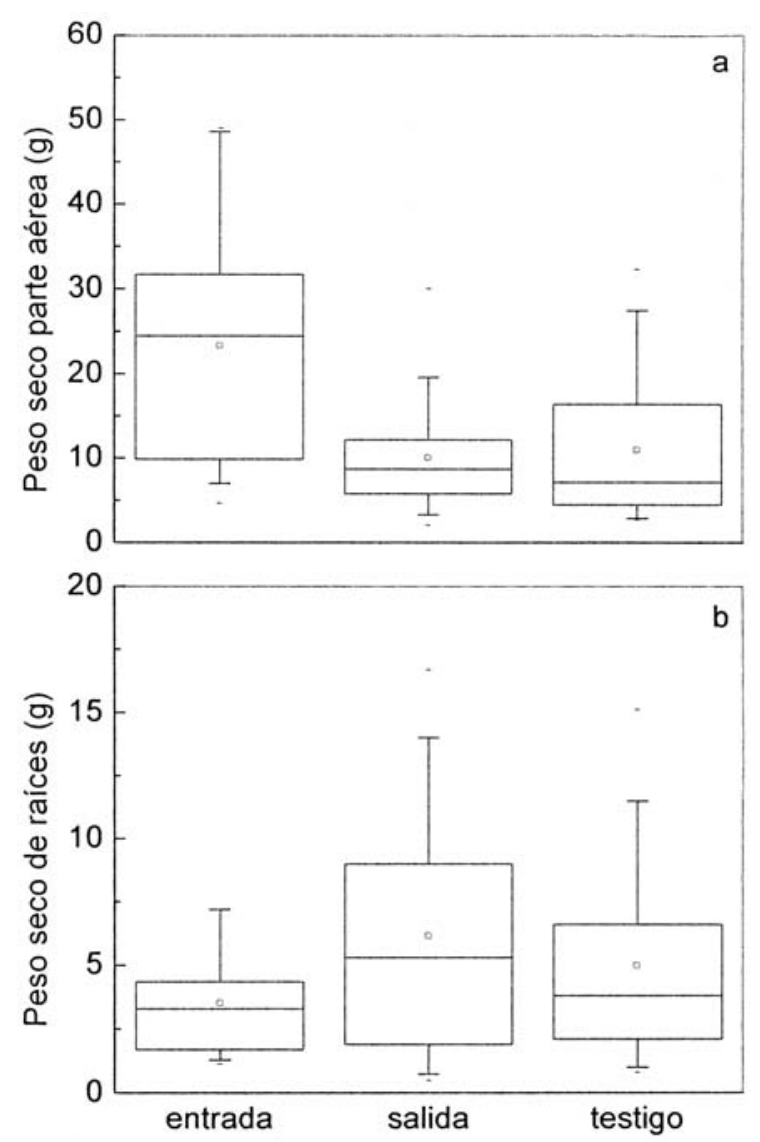

Figura 2. Gráficos de caja y bigote del peso seco de la parte aérea (a) y de las raíces (b) por planta de E. crassipes en los diferentes sitios de muestreo. Box and wisker plot of aerial part (a) and roots (b) dry weight per plant of E. crassipes in the different sampling sites.

La biomasa de raíces fue significativamente más alta en las plantas de la salida del humedal, en comparación con las de la entrada y testigo (Fig. 2b). No se observaron diferencias estadísticas entre las plantas de la entrada del humedal y las testigos.

La relación parte aérea/raíz disminuyó significativamente entre las plantas de la entrada respecto de las de la salida y testigo, probablemente debido a las diferentes concentraciones de fósforo. Los valores de mediana fueron: Entrada (6.8), Testigo (2.2), Salida (1.6); evidenciando un menor crecimiento radicular en la entrada del humedal.
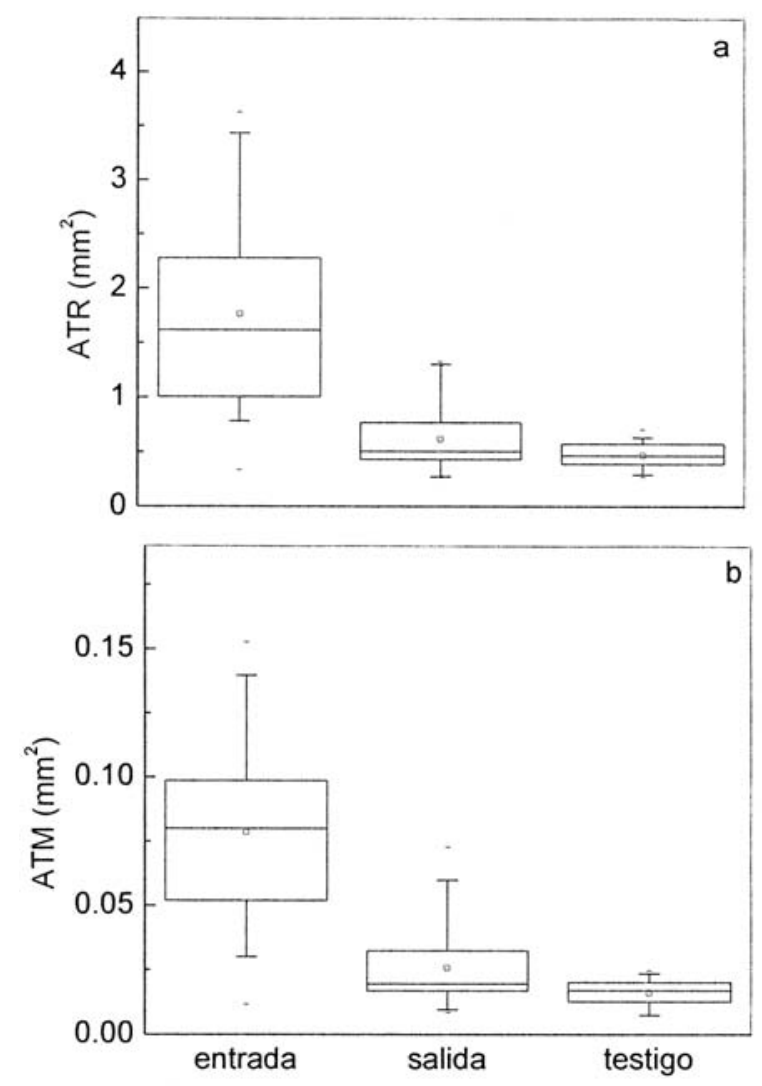

Figura 3. Gráficos de caja y bigote del área transversal de la raíz (ATR) (a) y de la médula (ATM) (b) de las raíces de $E$. crassipes en los diferentes sitios de muestreo. Box and wisker plot of the cross-sectional area of root (ATR) (a), and stele (ATM) (b), of E. crassipes roots in the different sampling sites.

\section{Morfología interna}

Las plantas de la entrada produjeron raíces más gruesas en comparación con las plantas de la salida y testigo. Se registró un área transversal de la raíz (ATR), área transversal de la médula (ATM), área transversal de los vasos metaxilemáticos tardíos (ATV) y área metaxilemática total (ATVt) mayor en las plantas de la entrada del humedal respecto a las de la salida y testigo (Figs. 3a, 3b, 4a y 4c, respectivamente). El número de vasos metaxilemáticos tardíos fue mayor en las plantas de la entrada del humedal en relación con las de la salida y testigo 
(Fig. 4b). En ningún parámetro morfológico interno de las raíces se encontraron diferencias significativas entre las plantas de la salida y testigo. En la Fig. 5 se muestran imágenes de
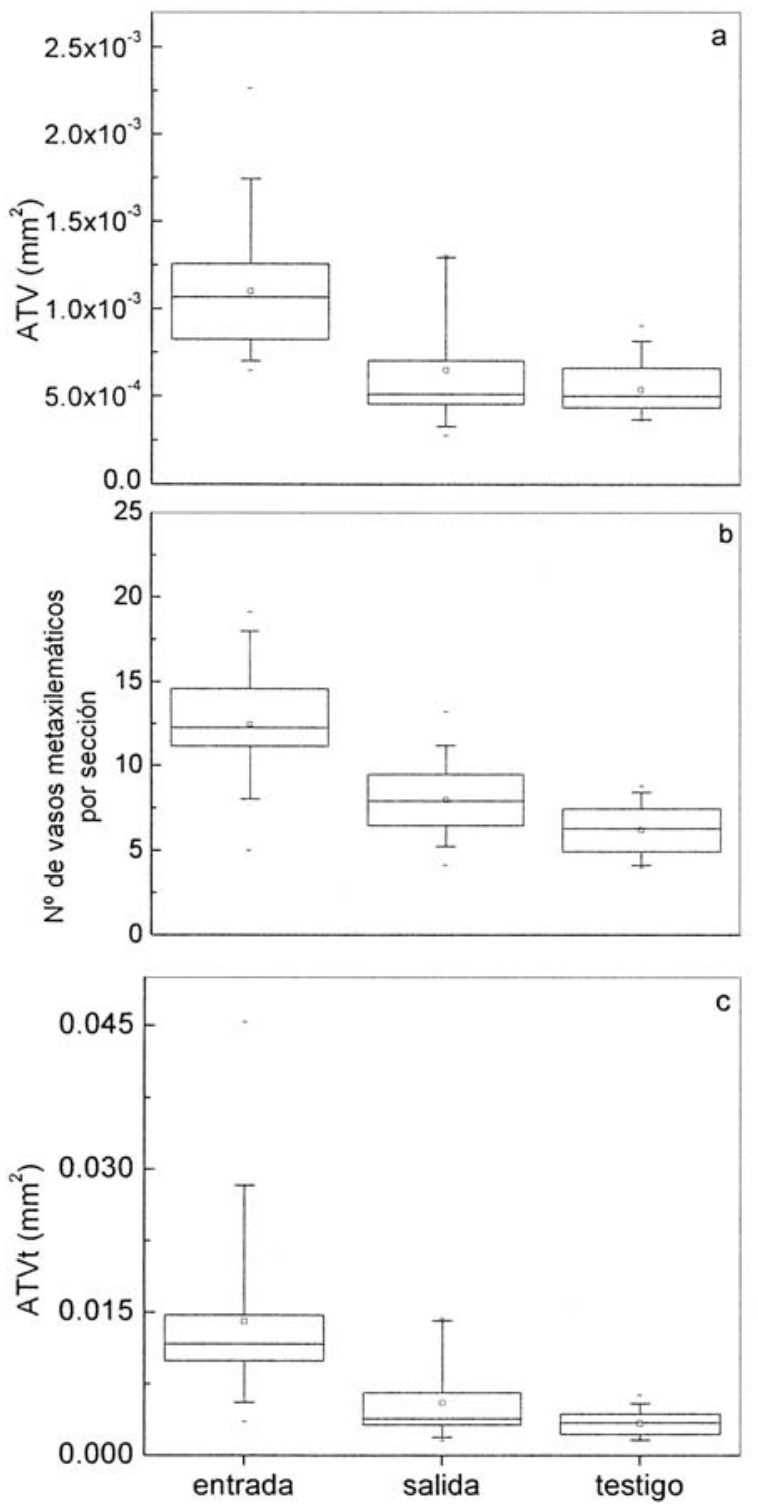

Figura 4. Gráficos de caja y bigote del área transversal de los vasos metaxilemáticos tardíos (ATV) (a), número de vasos metaxilemáticos por sección (b) y área total ocupada por el metaxilema (ATVt) (c) de las raíces de E. crassipes en los diferentes sitios de muestreo. Box and wisker plot of crosssectional area of the late metaxylematic vessels (ATV) (a), number of metaxylematic vessels per section (b) and total area ocupied by the metaxylem (ATVt) (c) of E. crassipes roots in the different sampling sites. microscopía óptica de las secciones transversales de las raíces de las plantas de la entrada, y salida del humedal artificial (Figs. 5a y 5b) y del humedal natural (Fig. 5c).

Se calculó la relación ATVt:ATR, dando como resultado 0.009 ( \pm 0.004$) ; 0.008( \pm 0.002)$ y $0.007( \pm 0.001)$, para la entrada, salida y testigo, respectivamente. No se registraron diferencias significativas entre estos valores, indicando que el área total del metaxilema (ATVt) aumentó al mismo tiempo que aumentó el área transversal de la raíz (ATR).

\section{DISCUSIÓN}

Ante un suministro alto de nutrientes, las plantas desarrollan mayormente la parte aérea, coincidiendo con los resultados obtenidos por otros autores (Reddy \& Sutton, 1984; Lallana \& Kieffer, 1988; Neiff et al., 2001). La mayor concentración de fósforo se presenta en la parte aérea, favoreciendo los procesos químicos inherentes a la fotosíntesis como la fotofosforilación y la reducción del NADP ${ }^{+}$a NADPH (Blanco, 1993).

A la salida del humedal, en donde el nutriente limitante fue el fósforo, de acuerdo a la relación $\mathrm{N}: \mathrm{P}$ obtenida (Tabla 1), E. crassipes asignó una mayor proporción de su biomasa a las raíces, coincidiendo con lo dicho por Bloom et al. (1985). El mayor desarrollo del sistema radicular de las plantas de la salida y testigo aumentó la capacidad de absorción de nutrientes al incrementarse la superficie de contacto raíz-agua. Esta estrategia permite a la planta sobrevivir en ambientes pobres en nutrientes (Xie \& Yu, 2003). También, las raíces de las plantas de la salida y testigo fueron más finas y largas, lo que concuerda con Ciro et al. (1999) y Xie \& Yu (2003), quienes expresan que las plantas con raíces finas y largas podrían ser más eficientes en la adquisición de $\mathrm{P}$ que aquellas plantas con raíces cortas y gruesas. Esto demuestra el alto grado de plasticidad morfológica del sistema radicular en plantas de crecimiento rápido como $E$. crassipes, en comparación con otras especies de crecimiento más lento (Robinson \& Van Vuuren, 1998). 

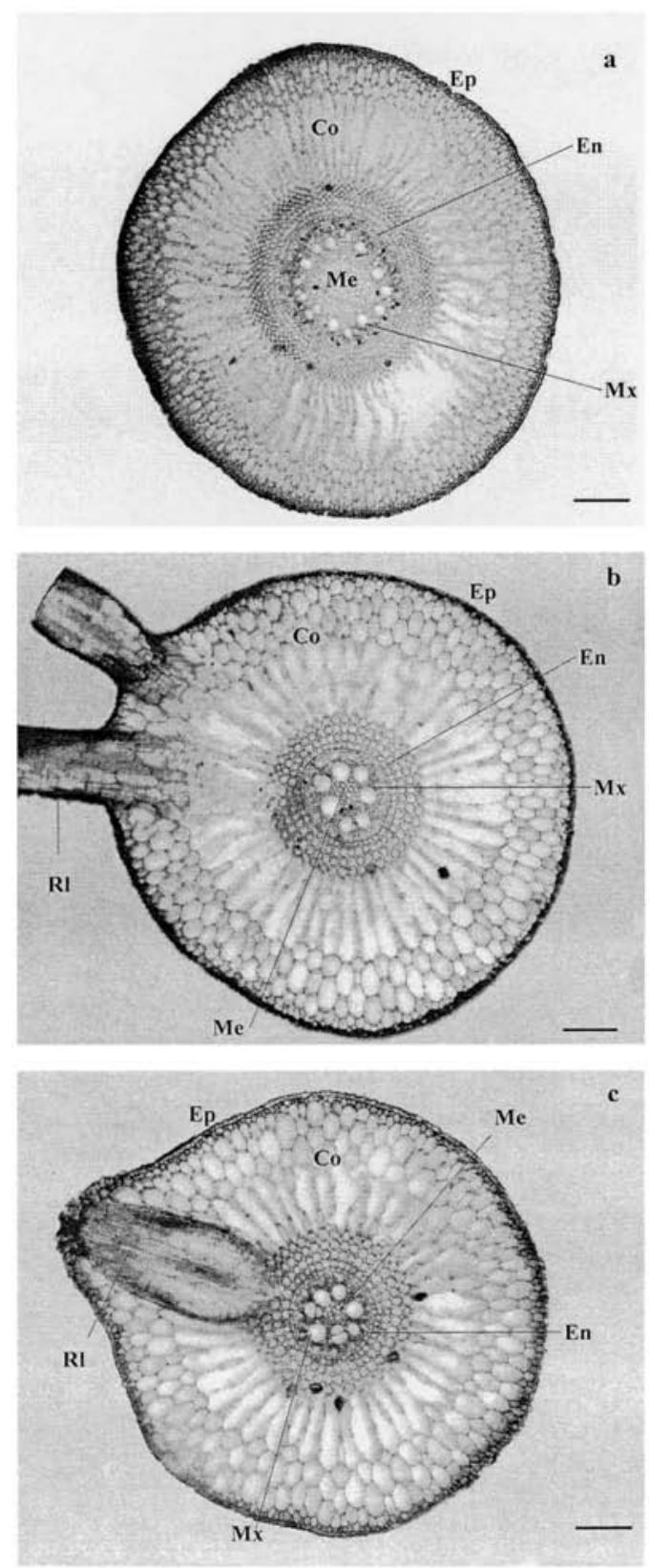

Figura 5. Imagen de microscopía óptica de las secciones transversales de las raíces de $E$. crassipes de la entrada (a) y salida (b) del humedal artificial, y del humedal natural testigo (c). Ep: epidermis, Co: cortex, En: endodermis, Me: Médula, Mx: vasos metaxilemáticos tardíos y R1: raíz lateral. Barra $=114 \mu \mathrm{m}$. Optical microscopy image of cross-sectional sections of E. crassipes roots from the inlet (a) and outlet (b) zones of the constructed wetland and from the control natural wetland (c). Ep: epidermis, Co: cortex, En: endodermis, Me: stele, Mx: large metaxylematic vessels and Rl: lateral roots. Bar $=114 \mu \mathrm{m}$.
Las raíces que crecen en un medio con alto contenido de nutrientes desarrollan vasos metaxilemáticos de mayor área. Un aumento del área metaxilemática a altas concentraciones de nutrientes, puede ser entendido en términos de la necesidad de aumentar su capacidad de transporte para un mayor desarrollo y mantenimiento de una mayor parte aérea (Wahl et al., 2001). Estudios realizados por Wahl et al. (2001) sostienen que las diferencias en las características anatómicas de raíces tratadas con diferentes concentraciones de nutrientes pueden ser explicadas por los efectos alométricos debidos a las diferencias en los tamaños de las plantas. En este trabajo, una mayor concentración de fósforo produjo un aumento en el área transversal de la raíz, a la vez que un aumento en los parámetros morfológicos internos. Este hecho se observó con las relaciones ATVt:ATR obtenidas.

Este estudio demostró que la plasticidad morfológica juega un rol importante en la adquisición de fósforo por $E$. crassipes en un ambiente heterogéneo. Los mecanismos que utiliza esta especie para sobrevivir en ambientes con diferentes concentraciones de fósforo, se basan en cambios en la capacidad de absorción a través de variaciones morfológicas del sistema radicular. Debido a las ventajas que produce una alta capacidad plástica, E. crassipes es una especie eficiente para ser utilizada en sistemas de tratamiento de efluentes.

\section{CONCLUSIONES}

Las diferentes concentraciones de fósforo presentes en el efluente cloacal, a la entrada y salida del humedal, produjeron variaciones en la morfología externa e interna de E. crassipes.

Las plantas de la entrada desarrollaron fundamentalmente la parte aérea, presentando una mayor altura y peso seco. Por el contrario, las plantas de la salida fueron más pequeñas pero desarrollaron más su sistema radicular con una mayor longitud y biomasa. Las raíces de $E$. crassipes de la salida del humedal fueron más finas y largas comparadas con las raí- 
ces cortas y gruesas de la entrada. Estos resultados verifican la influencia de los nutrientes en la morfología externa de la raíz, y sugieren que la plasticidad morfológica es el mecanismo más importante que posee la planta para adquirir nutrientes.

En las raíces de las plantas de la entrada se observó una mayor área de la médula, área y número de vasos metaxilemáticos y área metaxilemática total, comparadas con las plantas de la salida. Esta variación en los parámetros morfológicos internos se debió probablemente al aumento del área transversal de la raíz.

\section{BIBLIOGRAFÍA}

ADLER, P. R., S. T. SUMMERFELT, D. M. GLENN \& F. TAKEDA. 1996. Evaluation of a wetland system designed to meet stringent phosphorus discharge requirements. Water Environment Research, 68 (5): 836-840.

ANSOLA, G., J. M. GONZÁLEZ, R. CORTIJO \& E. DE LUIS. 2003. Experimental and full-scale pilot plant constructed wetlands for municipal wastewaters treatment. Ecological Engineering, 20: 4352.

APHA. 1998. Standard methods for the examination of water and wastewater. $20^{\text {th }}$ ed. USA: Amer. Publ. Health Assoc.

ATHIE, D. \& C. C. CERRI. 1987. The use of macrophytes in water pollution control. Pergamon Press. Oxford.

BATES, T. R. \& J. P. LYNCH. 1996. Stimulation of root hair elongation in Arabidopsis thaliana by low phosphorus availability. Plant Cell Environ, 19: 529-538.

BLANCO, A. 1993. Química biológica. El Ateneo. Buenos Aires. 677 pp.

BLOOM, A. J., F. S. CHAPIN \& H. A. MOONEY. 1985. Interpreting in plants - an economic analogy. Ann. Rev. Ecol. Syst., 16: 363 - 392.

BORCH, K., T. J. BOUMA, J. P. LYNCH \& K. M. BROWN. 1999. Ethylene: a regulator of root architectural responses to soil phosphorus availability. Plant Cell Environ, 22: 425-431.

CIRO, A. R., P. T. W. JOAO, V. SILVELENA \& J. R. VALDIR. 1999. The significance of root growth on cotton nutrition in an acidic low-P soil. Plant. Soil, 212: $185-190$.
D'AMBROGIO DE ARGÜESO, A. 1986. Manual de técnicas en histología vegetal. I-IV: 1-83. Hemisfero Sur. Buenos Aires. 83 pp.

DINKELAKER, B., B. HENGELER \& H. MARSHNER. 1995. Distribution and function of proteoid roots and other root clusters. Bot. Acta, 108: 183-200.

HARVEY, H. P. \& R. VAN DEN DRIESSCHE. 1999. Nitrogen and potassium effects on xylem cavitation and water - use efficiency in poplars. Tree Physiology, 19: 943-950.

HUTCHINGS, M. J. \& H. DE KROON. 1994. Foraging in plants: the role of morphological plasticity in resource acquisition. Adv. Ecol. Res., 26: $159-238$.

LALLANA, V. H. \& L. A. KIEFFER. 1988. Efecto del enriquecimiento de nutrientes en el crecimiento de Eichhornia crassipes (Mart.) SolmsLaubach "camalote". Rev. de la Asoc. de Cs. Nat. del Lit., 9 (2): 183-199.

LÓPEZ-BUCIO, J., E. HERNÁNDEZ-ABREU, L. SÁNCHEZ-CALDERÓN, M. F. NIETO-JACOBO, J. SIMPSON \& L. HERRERA-ESTRELLA. 2002. Phosphate availability alters architecture and causes changes in hormone sensitivity in the Arabidopsis root system. Plant Physiology, 129: 244-256.

MAINE, M. A., N. SUÑE, H. HADAD \& G. SÁNCHEZ. 2005. Spatial variation of phosphorus distribution in sediments of a constructed wetland. In: Phosphates in Sediments. Proceedings 4th International Symposium on Phosphate in Sediment .H. L. Golterman \& L. Serrano. (eds.).: 33-42. Backhuys Publishers. Leiden.

MAINE, M. A., N. SUÑE, H. HADAD, G. SÁNCHEZ \& C. BONETTO. 2004. Nutrient and metal removal in a constructed wetland for wastewater treatment from a metallurgic industry. Proceedings 9th International Conference on Wetlands Systems for Water Pollution Control (Avignon). International Water Association (IWA), Association Scientifique et technique pour l'eau et l'environnement (ASTEE). 2: 597-605.

MERLIN, G., J. L. PAJEAN \& T. LISSOLO. 2002. Performances of constructed wetlands for municipal wastewater treatment in rural mountainous area. Hydrobiologia, 469 (1-3): 87-98.

MURPHY, J. \& J. RILEY. 1962. A modified single solution method for determination of phosphate in natural waters. Anal. Chem. Acta, 27: 31-36.

NEIFF, J. J., A. POI DE NEIFF \& S. L. CASCO. 2001. The effect of extreme floods on Eichhornia 
crassipes (Mart. Solms) growth in Paraná River floodplain lakes. Acta Limnol. Bras., 13 (1): 5160.

PERDOMO, S., C. BANGUESES, J. FUENTES, J. CASTRO, S. ACEVEDO \& C. MICHELOTTI. 2000. Constructed wetlands: A more suitable alternative for wastewater purification in uruguayan dairy processing industry. Proceedings $7^{\text {th }}$ International Conference on Wetland Systems for Water Pollution Control (Florida). International Association on Water Quality (IAWQ). 1407-1415.

REDDY, K. R. \& D. L. SUTTON. 1984. Water hyacinths for water quality improvement and biomass production. J. Environ. Qual., 13: 1-7.

ROBINSON, P. \& M. M. VAN VUUREN. 1998. Responses of wild plants to nutrient patch in relation to growth rate and life form. In: Inherent variation in plant growth: Physiological mechanisms and ecological consequences. H. Lambers; H. Poorter \& M. M. Van Vuuren (eds.): 237 257. Backhuys Publishers. Leiden.

SAUNDERS, D. L. \& J. KALFF. 2001. Nitrogen retention in wetlands, lakes and rivers. Hydrobiologia, 443: 205-212.
SEDLACK, R. I. 1991. Phosphorus and nitrogen removal from municipal wastewater. $2^{\text {nd. }}$ ed. Chelsea, Mich.: Lewis Publishers. 256 pp.

VOLLENWEIDER, R. A. 1974. A manual on methods for measuring primary production in aquatic environments. IBP Handbook $\mathrm{N}^{\text {o }}$ 12. $2^{\text {nd. ed. Inter- }}$ national Biological Programme, Blackwell Scientific Publications. Oxford. 225 pp.

WAHL, S., P. RYSER \& P. J. EDWARDS. 2001. Phenotypic plasticity of grass root anatomy in response to light intensity and nutrient supply. Annals of Botany, 88: 1071-1078.

WALPOLE, R., R. MYERS \& S. MYERS. 1999. Probabilidad y estadística para ingenieros. $6^{\mathrm{a}}$. ed.: Prentice Hall Hispanoamericana. México. $752 \mathrm{pp}$.

WEISNER, S., P. ERIKSSON, W. GRANÉLI \& L. LEONARDSON. 1994. Influence of macrophytes on nitrate removal in wetlands. Ambio, 23 (6): 363-366.

XIE, Y. \& D. YU. 2003. The significance of lateral roots in phosphorus acquisition of water hyacinth (Eichhornia crassipes). Aquatic Botany, 75: 311321. 
